\title{
STRESSING ISSUE OF A PIEZOCERAMIC CANTILEVER WITH LONGITUDINAL POLARISATION AND ELECTRODE COATINGS
}

\author{
$U D C$ (621.315:MATLAB)
}

\section{Igor Jovanović ${ }^{1}$, Ljubiša Perić ${ }^{2}$, Uglješa Jovanović ${ }^{1}$, Dragan Mančić ${ }^{1}$ \\ ${ }^{1}$ University of Niš, Faculty of Electronic Engineering, Department of Electronics, Republic of Serbia \\ ${ }^{2}$ Regional Chamber of Economy Niš, Republic of Serbia}

\begin{abstract}
The main subject of this study is the investigation of the free vibration of a rectangular prismatic piezoceramic cantilever with longitudinal polarization and electrode coatings. Based on the general solution of coupled equations for piezoceramic material, applying the equations of electro-elasticity and satisfying electrical and mechanical conditions for the stress of a cantilever made from PZT4 piezoceramic material, componential displacements, electric potential, specific strain, electric field, and piezoelectric displacement, are determined and numerically obtained with Matlab software package. Based on the obtained equations and simulation results, it is possible to optimize the dimensions of the cantilever and determine the type of piezoceramic.
\end{abstract}

Key words: longitudinal polarization, piezoceramic cantilever, PZT4 piezoceramic material, Matlab

\section{INTRODUCTION}

Piezoceramic beam-like elements are the essential excitation elements in various ultrasonic devices (actuators and sensors). A beam with a fixed support at one end and without support at the other end is called a cantilever (or clamped-free) beam [1]. The ability to detect vibrations using the piezoelectric material has become a viable energy harvesting source [2]. Piezoelectric energy harvesting using cantilever-type structures has been extensively investigated due to its potential application in supplying power for wireless sensor networks [3]. The most commonly used piezoelectric materials are the following: Quartz, Polycrystalline ceramic, Lead Zirconate Titanate (PZT) [4].

Received Septembert 03, 2020

Corresponding author: Igor Jovanović

University of Niš, Faculty of Electronic Engineering, Aleksandra Medvedeva 14, 18000 Niš, Republic of Serbia

E-mail: igor.jovanovic@elfak.ni.ac.rs 
Piezoelectric devices are complex multi-physics systems requiring advanced methodologies to maximise their performance [5]. Problems related to the design of piezoelectric devices are power limitations due to the physical properties of the material from which the device is made, accurate determination of the resonant frequency, derivation of equations that describe the relationships between electromechanical quantities, etc. [6]. Vibrations modelling of a cantilever is studied theoretically in this paper so that the power conversion capability could be enhanced.

Knowing the natural resonant frequencies of piezoceramic elements is the initial condition when designing different devices [7]. A three-dimensional model is ideal for accurate analysis of these devices [8]. In the most general case, a complete three-dimensional analysis leads to a very complex system of nonlinear equations that are difficult to solve. For this reason, an effort has been made here to solve this problem by presenting an analysis of a twodimensional (2D) model of thin cantilevers in a Cartesian coordinate system.

In this paper, the general stress case of a rectangular prismatic piezoceramic cantilever with longitudinal polarization will be observed. The observed cantilever is loaded at the free end with a concentrated force. Besides, the cantilever also contains electrode coatings on the sides of $z= \pm h / 2$. It is assumed that an electric potential of $2 U_{0}$ is brought to the electrodes. Also, the assumption is that the influence of electromechanical characteristics of electrode coatings can be neglected. First, solutions for component displacements and electric potential in the polynomial form are assumed [9]. The mutually independent coefficients are determined from the given boundary conditions on the surfaces of a rectangular piezoceramic body. Then, the exact solutions for the component displacements and electric potential are determined. Afterwards, using Cauchy's kinematic equations, one may obtain solutions for component mechanical deformations: dilatation and slide.

\section{FUNDAMENTAL EQUATIONS}

In the observed case, if a constant concentrated force $\vec{F}$ is applied, as shown in Fig. 1 , there is only the occurrence of normal mechanical stress $\sigma_{x}$ in the axial direction (along the $x$-axis) and tangential mechanical stress in the cross-sectional plane $\tau_{x z}$ aimed in the direction of the force $\vec{F}$.

The vector of the mechanical displacement components is represented by the projections of the displacement vector (through the Cartesian components of the displacement vectors) $u, v$ and $w: \vec{s}=u(x, y, z) \vec{i}+v(x, y, z) \vec{j}+w(x, y, z) \vec{k}$. Meanwhile, volumetric forces are neglected [10]. Also, the assumption was introduced that the mechanical displacement components $u$ and $w$ are independent of the $y$ coordinate, i.e.:

$$
u=u(x, z), w=w(x, z),
$$

while the component of the piezoelectric displacement vector in the $y$-axis direction is equal to zero:

$$
D_{y}=0
$$

The boundary conditions on the sides where the electrode coatings are $(z= \pm h / 2)$ can be expressed as follows [11]: 


$$
\left.\sigma_{z}\right|_{z= \pm h / 2}=0,\left.\tau_{x z}\right|_{z= \pm h / 2}=0,\left.\quad \psi\right|_{z= \pm h / 2}= \pm U_{0},
$$

where $\psi$ is an electric potential.

If the cross-sectional dimensions of the rectangular cantilever are small compared to the length $l$, the boundary conditions (3) are fulfilled using expressions from the reference [11], where the mechanical stress components for the observed case are:

$$
\sigma_{x}=-\frac{F}{I_{y}} x z, \quad \tau_{x z}=-\frac{F}{16 I_{y}}\left(h^{2}-4 z^{2}\right),
$$

where $I_{y}=b h^{3} / 12$ is the axial moment of inertia for surfaces parallel to the front $x=0$.

The following integral conditions are applied on the surface $x=0$ :

$$
\left.\sigma_{x}\right|_{x=0}=0,-\left.2 b \int_{-h / 2}^{h / 2} \tau_{x z}\right|_{x=0} d z=F .
$$

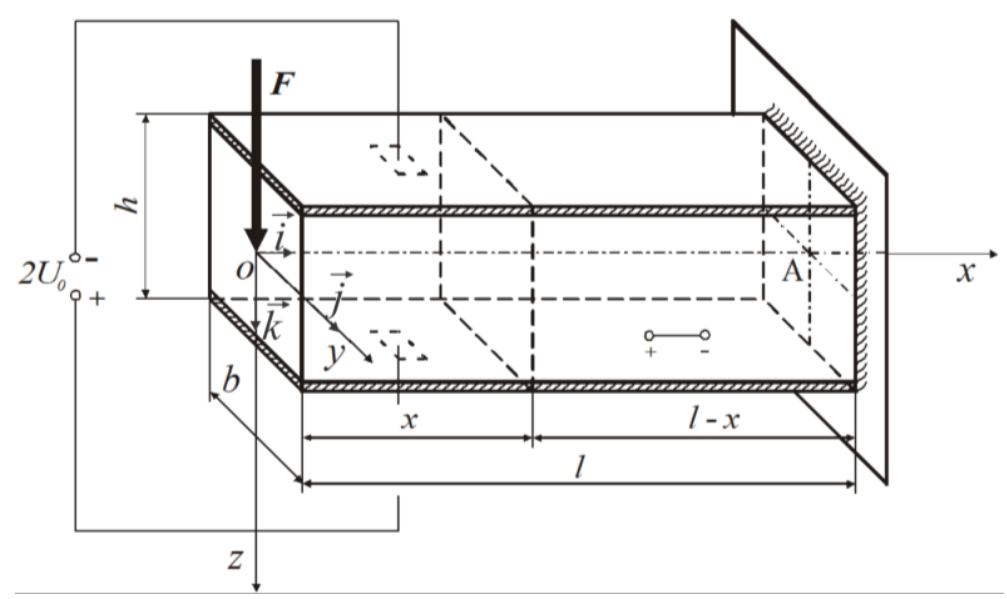

Fig. 1 Stressing of piezoceramic cantilever with longitudinal polarisation and electrodes

The conditions for rigid clamping of the cantilever for the front surface $x=l$ are:

$$
w(l, 0)=0,\left.\frac{\partial w}{\partial z}\right|_{\substack{x=l \\ z=0}}=0 .
$$

If the displacement vector $\vec{s}$ components $u$ and $w$, as well as the electric potential $\psi$, are represented in the polynomial form [12], the following expressions are obtained:

$$
\begin{gathered}
u=a_{2} z+a_{4} x^{2} z, \\
w=b_{0}+b_{2} x+b_{3} x^{3}, \\
\psi=c_{1} z+c_{4} z^{3} .
\end{gathered}
$$


If the equations of electrostatics:

$$
\operatorname{div} \vec{D}=\frac{\partial D_{x}}{\partial x}+\frac{\partial D_{y}}{\partial y}+\frac{\partial D_{z}}{\partial z}=0, \vec{E}=-\operatorname{grad} \psi=-\left(\frac{\partial \psi}{\partial x} \vec{i}+\frac{\partial \psi}{\partial y} \vec{j}+\frac{\partial \psi}{\partial z} \vec{k}\right)
$$

as well as Cauchy's kinematic equations:

$$
\begin{gathered}
\varepsilon_{x}=\frac{\partial u}{\partial x}, \quad \varepsilon_{y}=\frac{\partial v}{\partial y}, \\
\varepsilon_{z}=\frac{\partial w}{\partial z}, \gamma_{x y}=\frac{\partial u}{\partial y}+\frac{\partial v}{\partial x}, \\
\gamma_{x z}=\frac{\partial u}{\partial z}+\frac{\partial w}{\partial x}, \quad \gamma_{y z}=\frac{\partial v}{\partial z}+\frac{\partial w}{\partial y},
\end{gathered}
$$

are applied in expression (7), the following system of equations is obtained:

$$
\begin{gathered}
E_{x}=0, \quad E_{y}=0, \\
E_{z}=-c_{1}-3 c_{4} z^{2}, \\
\varepsilon_{x}=2 a_{4} x z, \\
\gamma_{x z}=a_{2}+b_{2}+\left(a_{4}+3 b_{3}\right) x^{2}, \\
\varepsilon_{y}=0, \quad \varepsilon_{z}=0, \gamma_{x y}=0, \gamma_{y z}=0,
\end{gathered}
$$

where $a_{2}, a_{4}, b_{0}, b_{2}, b_{3}, c_{1}$ and $c_{4}$ are newly introduced coefficients, $\varepsilon_{x}, \varepsilon_{y}$ and $\varepsilon_{z}$ are dilatation components, $\gamma_{x y}, \gamma_{x z}$ and $\gamma_{y z}$ are slide components, $E_{x}, E_{y}$ and $E_{z}$ are the electric field vector $(\vec{E})$ components. Given the initial assumption that for the observed case of a cantilever loaded at the free end with concentrated force, there is only the occurrence of normal mechanical stress $\sigma_{x}$, and tangential mechanical stress $\tau_{x z}$, and mechanical displacement component $v$ satisfy the following relations $\partial v / \partial x=\partial v / \partial y=\partial v / \partial z=0$.

For the adopted longitudinal polarization, equation which describes the mutual coupling between electrical and mechanical variables has the following form:

$$
\begin{gathered}
\varepsilon_{x}=\varepsilon_{33}^{E} \sigma_{x}+\varepsilon_{13}^{E}\left(\sigma_{y}+\sigma_{z}\right)+b_{33} E_{x}, \\
\varepsilon_{y}=\varepsilon_{13}^{E} \sigma_{x}+\varepsilon_{11}^{E} \sigma_{y}+\varepsilon_{12}^{E} \sigma_{z}+b_{31} E_{x}, \\
\varepsilon_{z}=\varepsilon_{13}^{E} \sigma_{x}+\varepsilon_{12}^{E} \sigma_{y}+\varepsilon_{11}^{E} \sigma_{z}+b_{31} E_{x}, \\
\gamma_{x y}=\varepsilon_{44}^{E} \tau_{x y}+b_{15} E_{y}, \\
\gamma_{x z}=\varepsilon_{44}^{E} \tau_{x z}+b_{15} E_{z}, \\
\gamma_{y z}=2\left(\varepsilon_{11}^{E}-\varepsilon_{12}^{E}\right) \tau_{y z}, \\
D_{x}=d_{33}^{\sigma} E_{x}+b_{33} \sigma_{x}+b_{31}\left(\sigma_{y}+\sigma_{z}\right), \\
D_{y}=d_{11}^{\sigma} E_{y}+b_{15} \tau_{x y}, \\
D_{z}=d_{11}^{\sigma} E_{z}+b_{15} \tau_{x z} .
\end{gathered}
$$


where $D_{x}, D_{y}$ and $D_{z}\left[\mathrm{C} / \mathrm{m}^{2}\right]$ are piezoelectric displacement vector components, $\varepsilon_{11}{ }^{E}, \varepsilon_{12}{ }^{E}$, $\varepsilon_{13}{ }^{E}, \varepsilon_{33}{ }^{E}$, and $\varepsilon_{44}{ }^{E}\left[\mathrm{~m}^{2} / \mathrm{N}\right]$ are coefficients of elastic power at a given (zero) electric field, $b_{31}, b_{15}$, and $b_{33}[\mathrm{C} / \mathrm{N}]$ are piezoelectric constants (piezomodules), $d_{11}{ }^{\sigma}$ and $d_{33^{\sigma}}{ }^{\sigma}[\mathrm{F} / \mathrm{m}]$ are dielectric permeabilities at a given zero mechanical stress.

Seven unknown coefficients: $a_{2}, a_{4}, b_{0}, b_{2}, b_{3}, c_{1}$, and $c_{4}$, which enter into expressions (7) and (10), have to be determined to fulfil the system of equations of electro-elasticity (11) and boundary conditions (3), (5), and (6):

$$
\begin{gathered}
2 a_{4} x z=-\frac{F \varepsilon_{33}^{E}}{I_{y}} x z, \\
a_{2}+b_{2}+\left(a_{4}+3 b_{3}\right) x^{2}=-\frac{F \varepsilon_{44}^{E}}{8 I_{y}} h^{2}+\frac{F \varepsilon_{44}^{E}}{2 I_{y}} z^{2}-b_{15} c_{1}-3 c_{4} b_{15} z^{2}, \\
D_{x}=-\frac{F b_{33}}{I_{y}} x z, \quad D_{z}=-d_{11}^{\sigma} c_{1}-\frac{F b_{15}}{2 I_{y}} \frac{h^{2}}{4}+\left(\frac{F b_{15}}{2 I_{y}}-3 d_{11}^{\sigma} c_{4}\right) z^{2}, \\
-b_{33} \frac{F}{I_{y}} z+\left.2\left(\frac{F b_{15}}{2 I_{y}}-3 d_{11}^{\sigma} c_{4}\right) z\right|_{d i v D=0}=0, \\
c_{1} \frac{h}{2}+\left.c_{4} \frac{h^{3}}{8}\right|_{z=\frac{\mathrm{h}}{2}}=U_{0}, \quad \\
-c_{1} \frac{h}{2}+\left.c_{4} \frac{h^{3}}{8}\right|_{z=-\frac{\mathrm{h}}{2}}=-U_{0}, \\
\left.w\right|_{\substack{z=0 \\
x=l}}=b_{0}+b_{2} l+b_{3} l^{3}=0,\left.\quad \frac{\partial w}{\partial x}\right|_{\substack{z=0 \\
x=l}}=b_{2}+3 b_{3} l^{2}=0 .
\end{gathered}
$$

From the system of equations (12) unknown coefficients are determined as:

$$
\begin{gathered}
a_{2}=\frac{F \varepsilon_{33}^{E}}{2 I_{y}} l^{2}-\frac{2 U_{0}}{h} b_{15}-\frac{F \varepsilon_{44}^{E}}{3 I_{y}} \frac{h^{2}}{4}, a_{4}=-\frac{F \varepsilon_{33}^{E}}{2 I_{y}}, \\
b_{0}=\frac{F \varepsilon_{33}^{E}}{3 I_{y}} l^{3}, b_{2}=-\frac{F \varepsilon_{33}^{E}}{2 I_{y}} l^{2}, \quad b_{3}=\frac{F \varepsilon_{33}^{E}}{6 I_{y}}, \\
c_{1}=\frac{2 U_{0}}{h}-\frac{F}{6 I_{y}} \frac{b_{15}-b_{33}}{d_{11}^{\sigma}} \frac{h^{2}}{4}, \quad c_{4}=\frac{F}{6 I_{y}} \frac{b_{15}-b_{33}}{d_{11}^{\sigma}} .
\end{gathered}
$$

By introducing the obtained values for coefficients (13) into expressions (7) and (10), one gets solutions for componential displacements of the displacement vector $\vec{s}$, electric potential $\psi$, specific strains (dilatation $\varepsilon_{x}$ and slide $\gamma_{x z}$ ), electric field $E_{z}$, and piezoelectric displacements $D_{x}$ and $D_{z}$, for the rectangular prismatic cantilever with longitudinal polarisation and electrode coatings on the sides $z= \pm h / 2$, in the form of:

$$
u=\left(\frac{F \varepsilon_{33}^{E}}{2 I_{y}} l^{2}-\frac{2 U_{0}}{h} b_{15}-\frac{F \varepsilon_{44}^{E}}{3 I_{y}} \frac{h^{2}}{4}\right) z-\frac{F \varepsilon_{33}^{E}}{2 I_{y}} x^{2} z
$$




$$
\begin{gathered}
w=\frac{F \varepsilon_{33}^{E}}{3 I_{y}} l^{3}-\frac{F \varepsilon_{33}^{E}}{2 I_{y}} l^{2} x+\frac{F \varepsilon_{33}^{E}}{6 I_{y}} x^{3}, \\
\psi=\left(\frac{2 U_{0}}{h}-\frac{F}{6 I_{y}} \frac{b_{15}-b_{33}}{d_{11}^{\sigma}} \frac{h^{2}}{4}\right) z+\frac{F}{6 I_{y}} \frac{b_{15}-b_{33}}{d_{11}^{\sigma}} z^{3}, \\
\varepsilon_{x}=-\frac{F \varepsilon_{33}^{E}}{I_{y}} x z, \\
\gamma_{x z}=-\frac{2 U_{0}}{h} b_{15}-\frac{F \varepsilon_{44}^{E}}{3 I_{y}} \frac{h^{2}}{4}, \\
E_{z}=-\frac{2 U_{0}}{h}+\frac{F}{2 I_{y}} \frac{b_{15}-b_{33}}{d_{11}^{\sigma}}\left(\frac{h^{2}}{12}-z^{2}\right) . \\
D_{x}=-b_{33} \frac{F}{I_{y}} x z . \\
D_{z}=-\frac{2 U_{0}}{h} d_{11}^{\sigma}-\frac{F}{6 I_{y}}\left(2 b_{15}+b_{33}\right) \frac{h^{2}}{4}+\frac{F b_{33}}{2 I_{y}} z^{2} .
\end{gathered}
$$

\section{NUMERICAL ANALYSIS}

The subject of observation in this paper is stressing of a piezoceramic PZT4 [13] cantilever with a longitudinal type of polarization and electrode coatings, with the following dimensions: $b=4.1 \mathrm{~mm}, h=20.1 \mathrm{~mm}, l=30.1 \mathrm{~mm}$ and density $\rho=7500 \mathrm{~kg} / \mathrm{m}^{3}$, loaded by the concentrated force (see Fig. 1). Material coefficients used in the analysis are listed in Table 1.

Table 1 Design parameters of the piezoceramic cantilever

\begin{tabular}{cc}
\hline Symbol & Value \\
\hline$\varepsilon_{13}{ }^{E}$ & $-5.31 \cdot 10^{-12}$ \\
$\varepsilon_{33}{ }^{2}$ & $15.5 \cdot 10^{-12}$ \\
$\varepsilon_{44}{ }^{E}$ & $39 \cdot 10^{-12}$ \\
$b_{31}$ & $-123 \cdot 10^{-12} \mathrm{~m} / \mathrm{V}$ \\
$b_{33}$ & $289 \cdot 10^{-12} \mathrm{~m} / \mathrm{V}$ \\
$b_{15}$ & $496 \cdot 10^{-12} \mathrm{~m} / \mathrm{V}$ \\
$\varepsilon_{0}$ & $8.854 \cdot 10^{-12} \mathrm{~F} / \mathrm{m}$ \\
$d_{11}$ & $1475 \cdot \varepsilon_{0} \mathrm{~F} / \mathrm{m}$ \\
$d_{33}$ & $1300 \cdot \varepsilon_{0} \mathrm{~F} / \mathrm{m}$ \\
\hline
\end{tabular}

Based on equations (14)-(21) a numerical analysis was performed using Matlab software, and bi-parametric surfaces of state were obtained for: componential displacement $u=u(x, z)$ (see Fig. 2); componential displacement $w=w(x, F)$ (see Fig. 3); electric potential $\psi=\psi(z, F)$ (see Fig. 4); specific strain - dilatation $\varepsilon_{x}=\varepsilon_{x}(x, z)$ (see Fig. 5); specific strain - slide $\gamma_{x y}=$ $\gamma_{x y}\left(U_{0}, F\right)$ (see Fig. 6); electric field $E_{z}=E_{z}\left(z, F, U_{0}\right)$ (see Figs. 7 and 8); piezoelectric displacement $D_{x}=D_{x}(x, z)$ (see Fig. 9); and piezoelectric displacement $D_{z}=D_{z}(z, F)$ (see Fig. 10). 


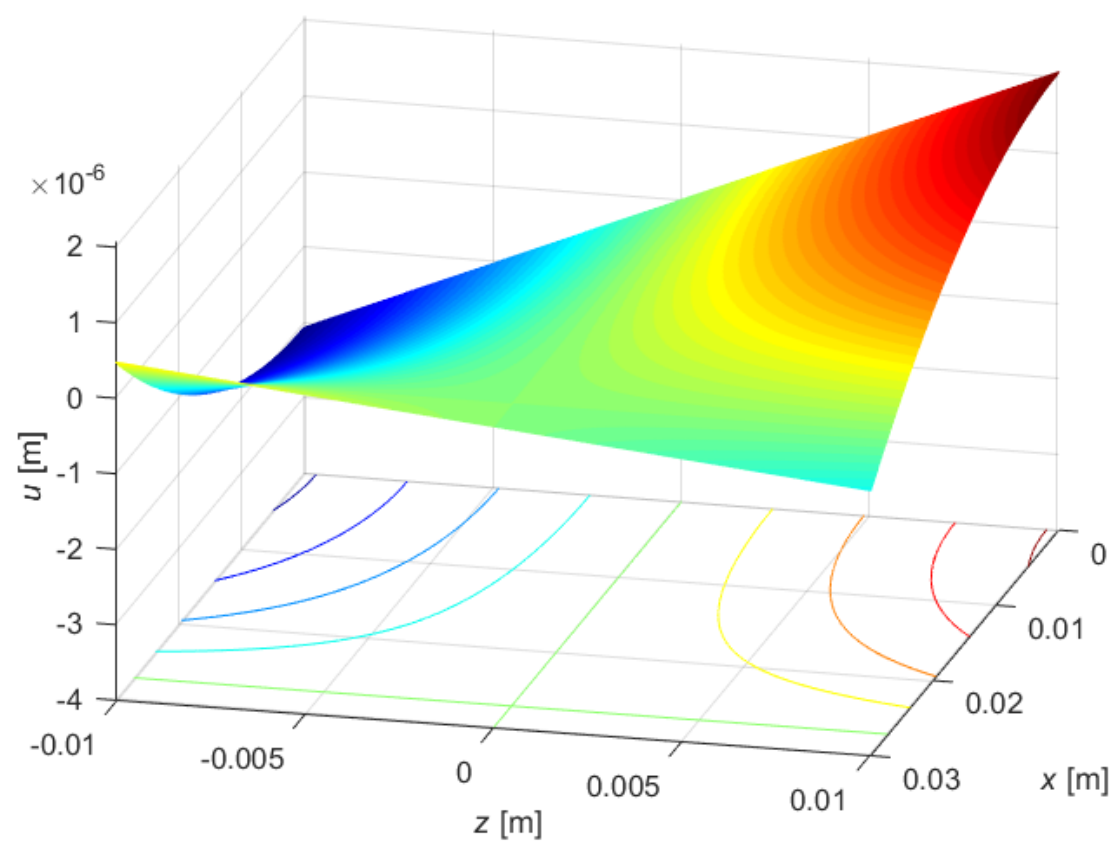

Fig. 2 Componential displacement $u=u(x, z)$

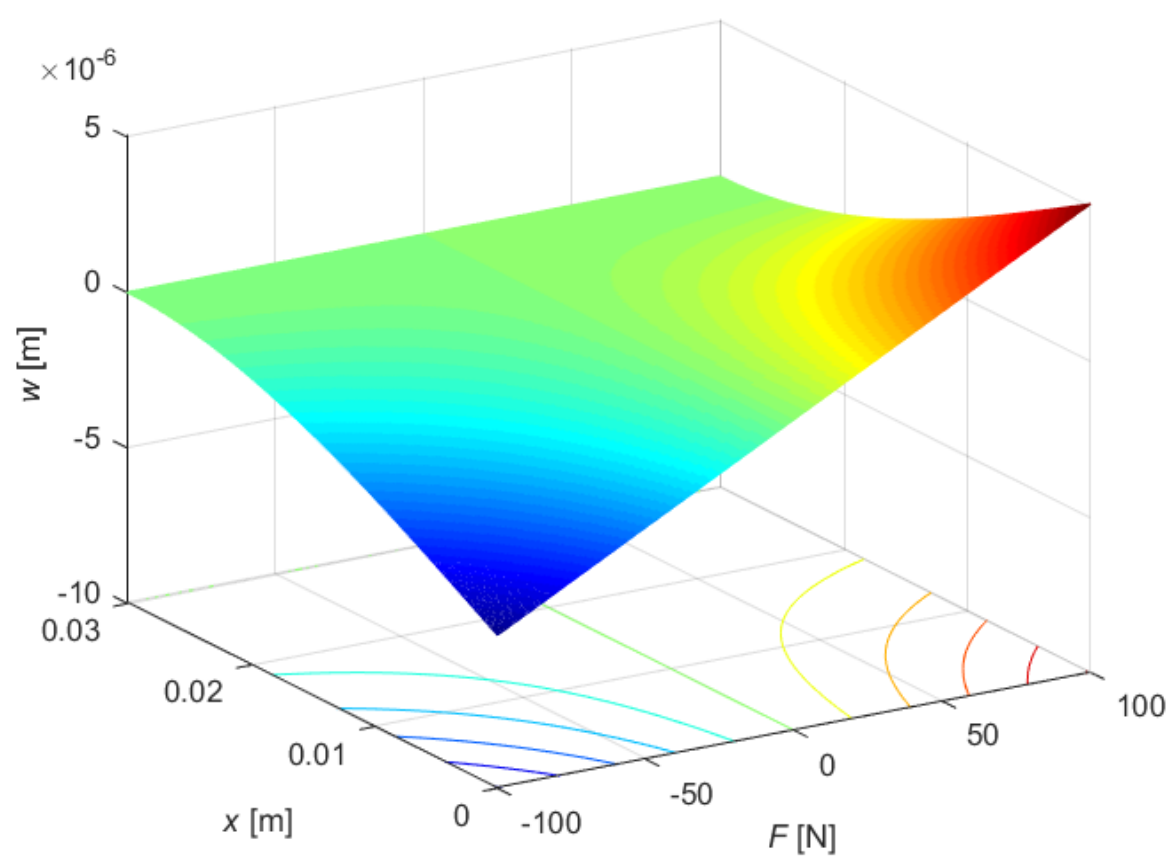

Fig. 3 Componential displacement $w=w(x, F)$ 


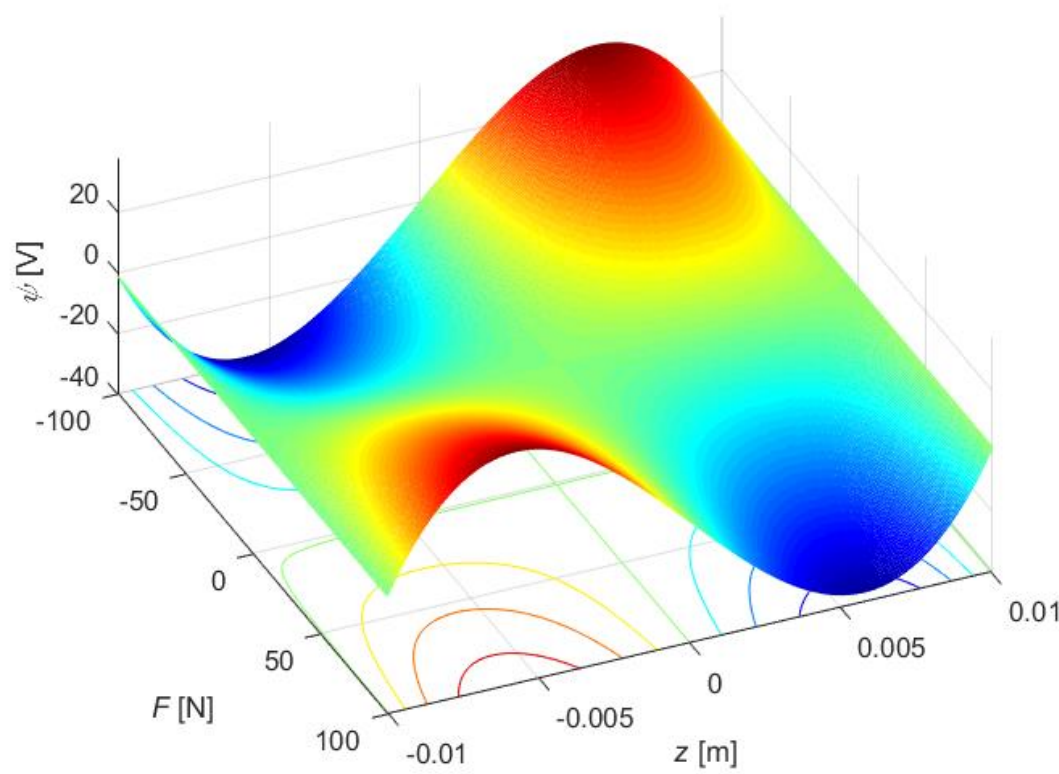

Fig. 4 Electric potential $\psi=\psi(z, F)$

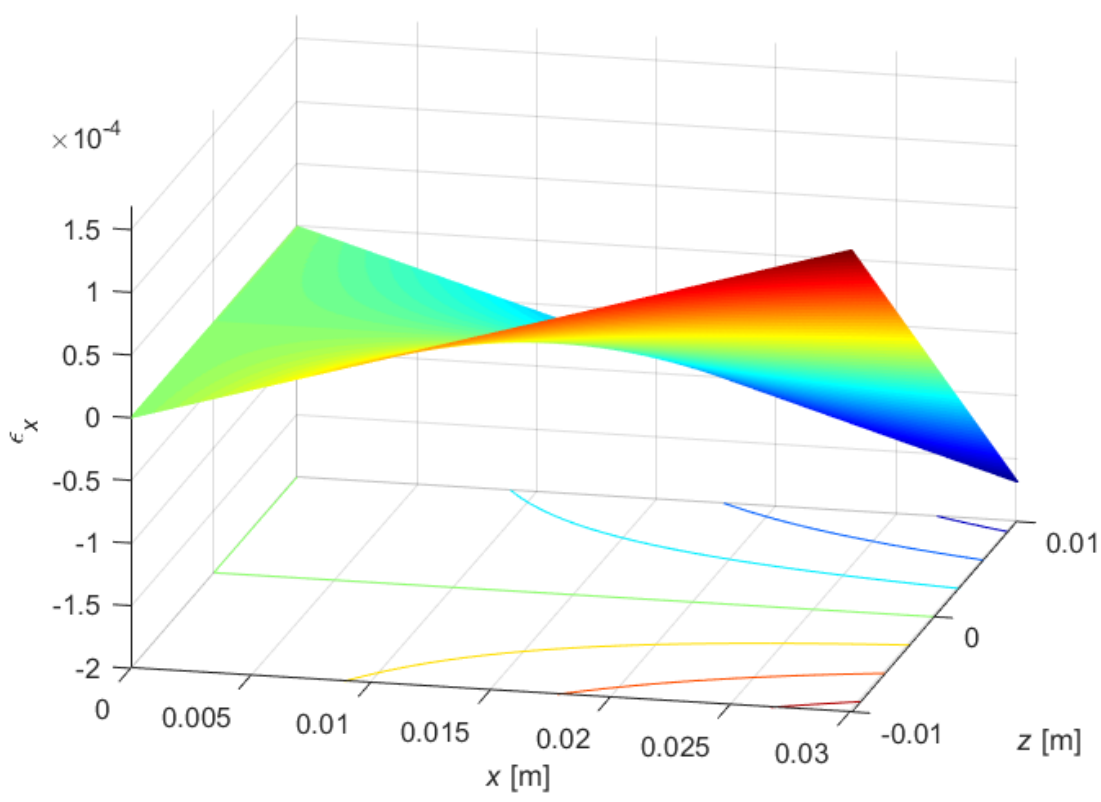

Fig. 5 Specific strain - dilatation $\varepsilon_{x}=\varepsilon_{x}(x, z)$ 


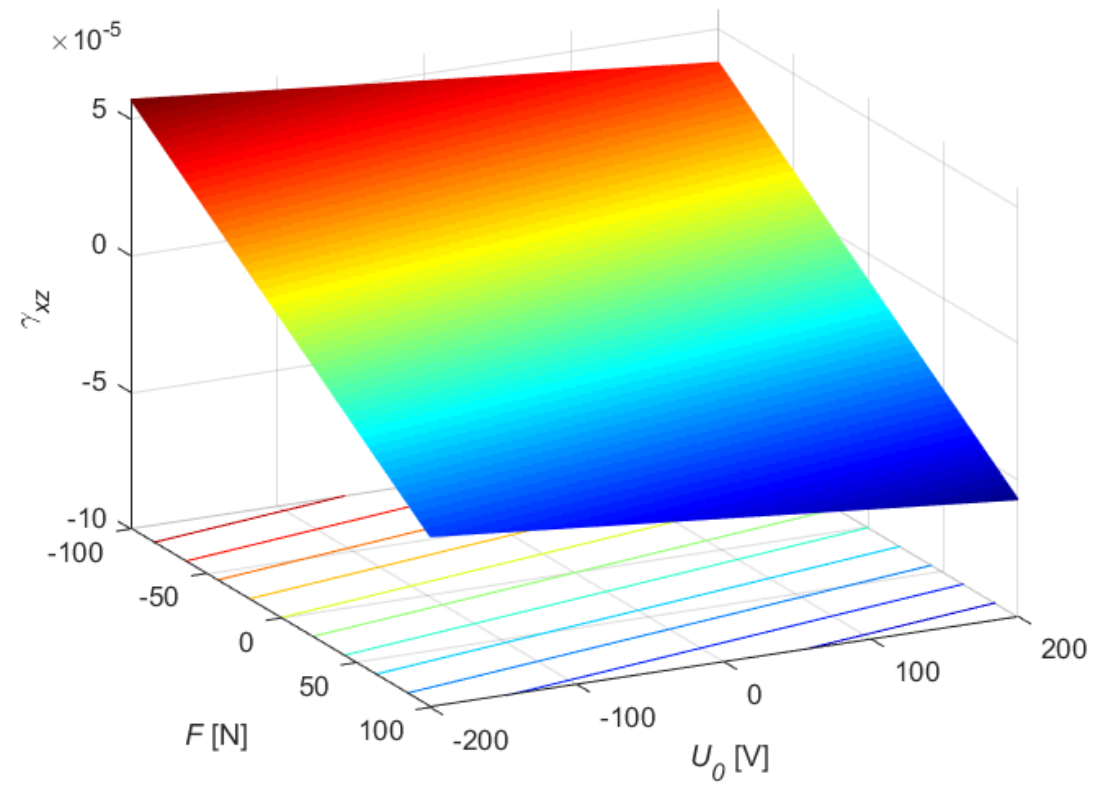

Fig. 6 Specific strain - slide $\gamma_{x y}=\gamma_{x y}\left(U_{0}, F\right)$

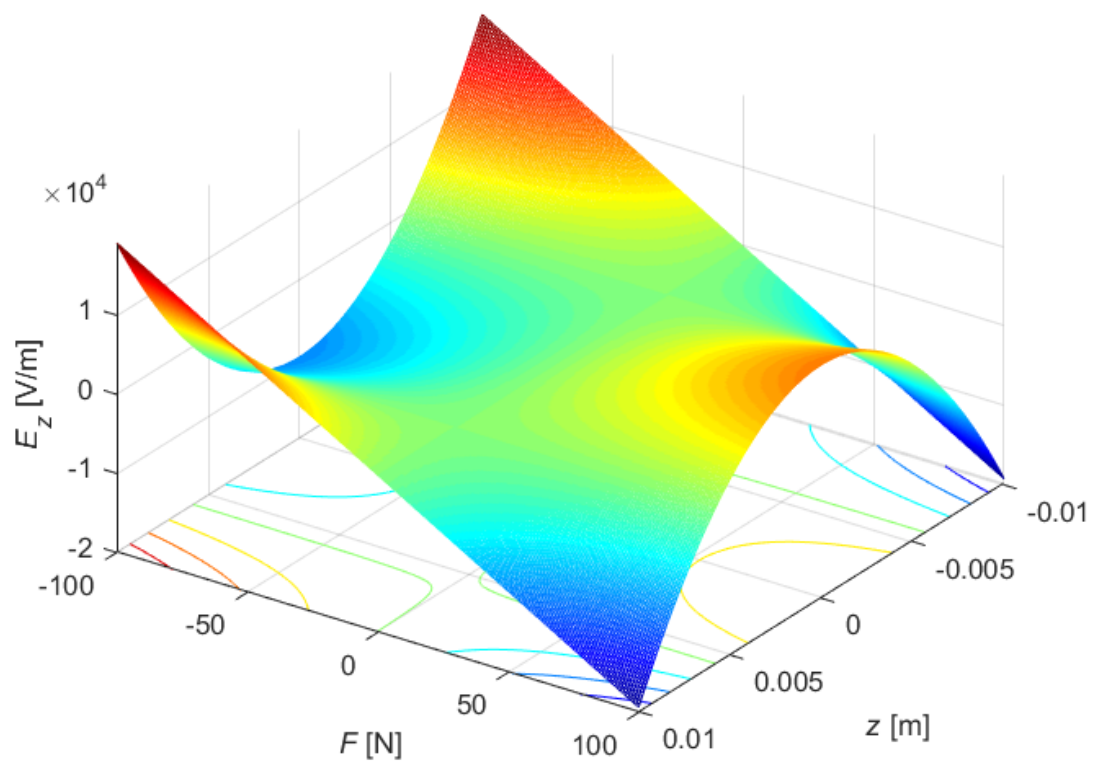

Fig. 7 Electric field $E_{z}=E_{z}(z, F)$ 


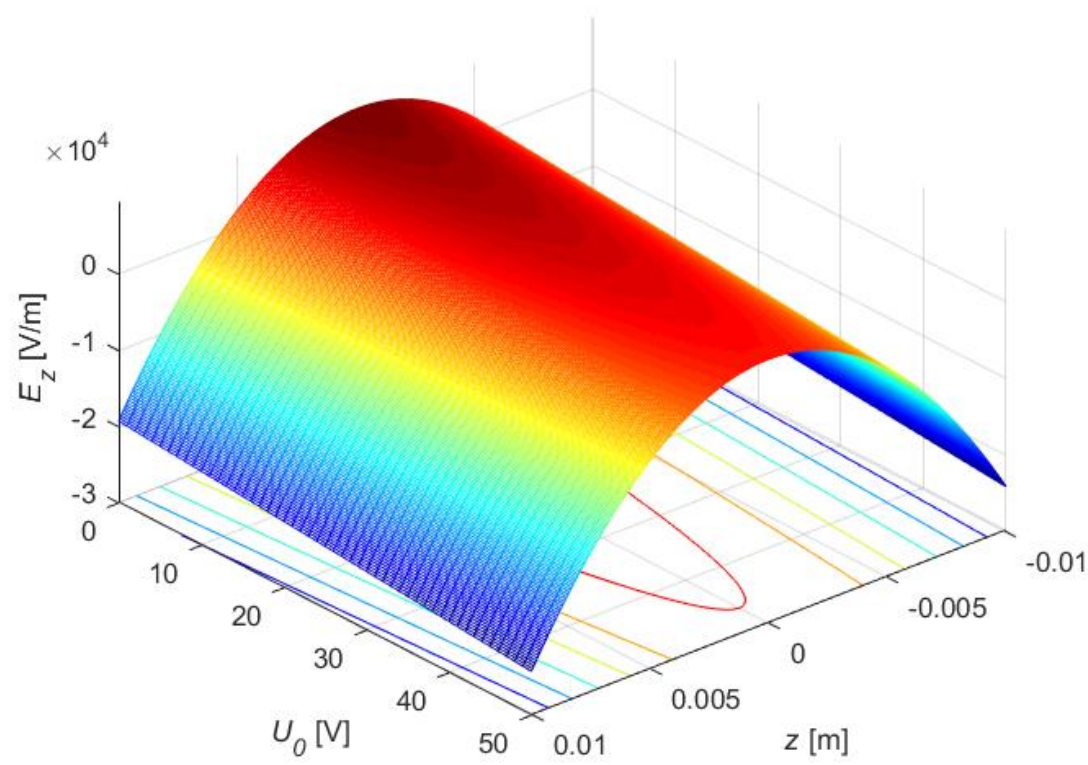

Fig. 8 Electric field $E_{z}=E_{z}\left(z, U_{0}\right)$

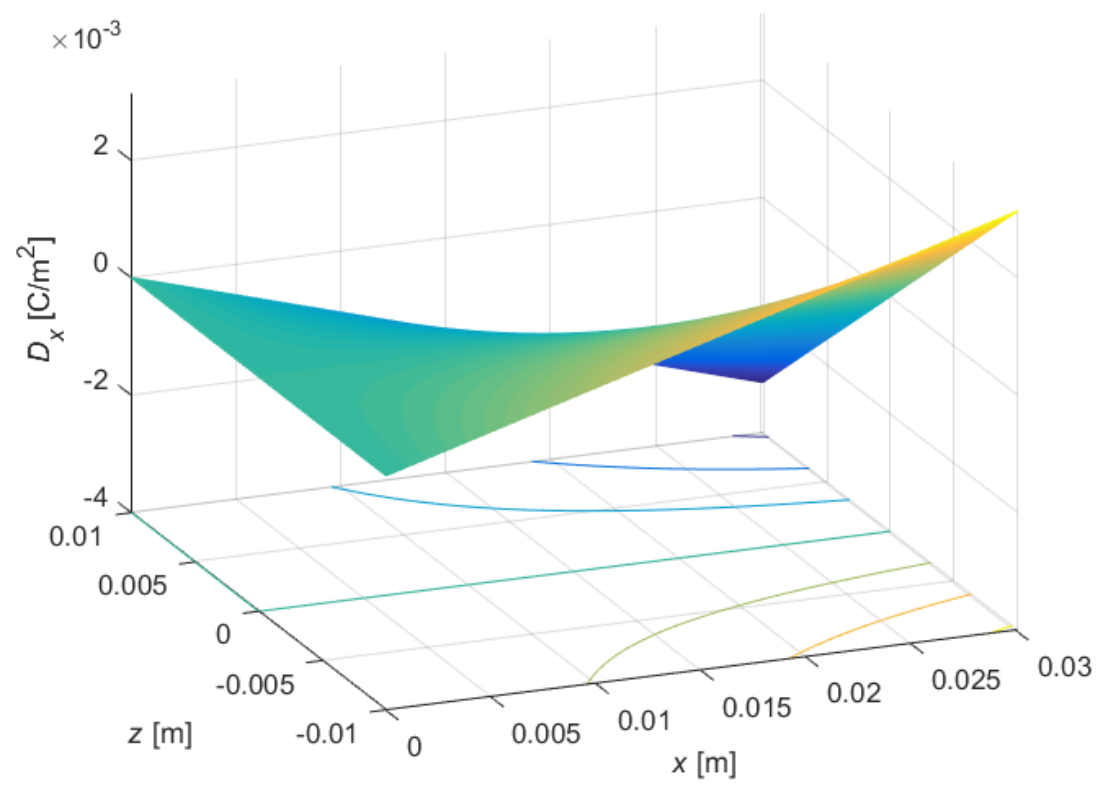

Fig. 9 Piezoelectric displacement $D_{x}=D_{x}(x, z)$ 


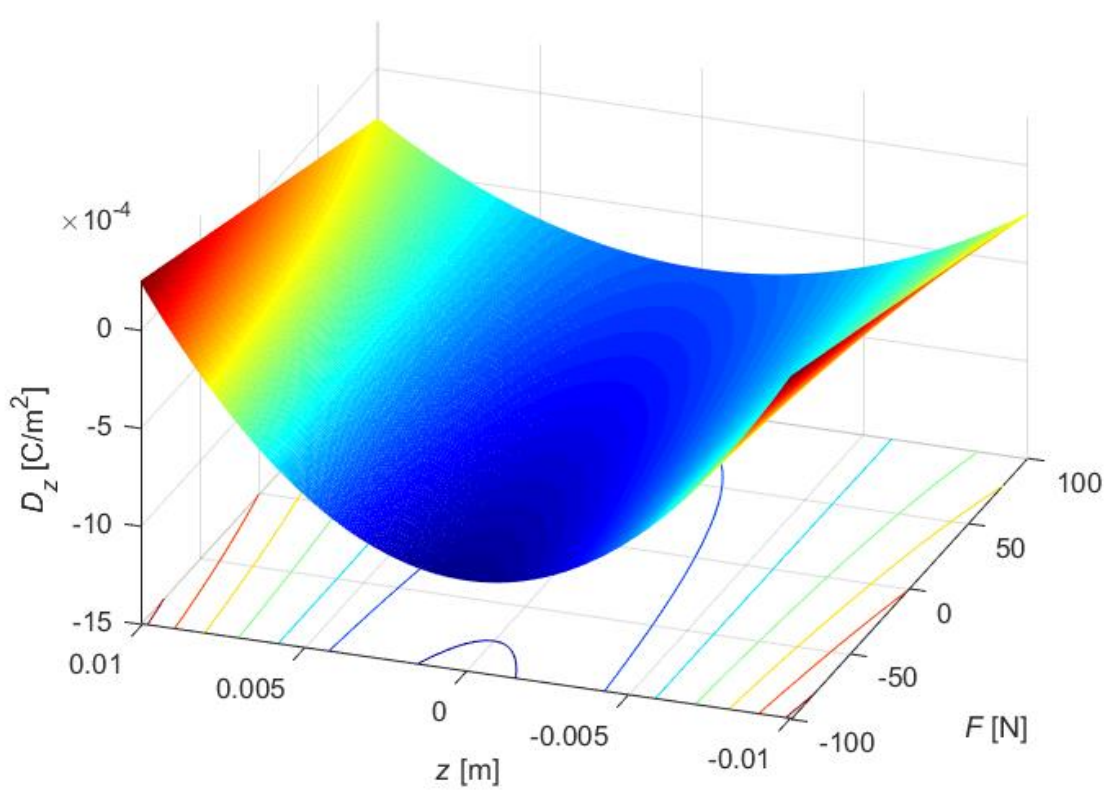

Fig. 10 Piezoelectric displacement $D_{z}=D_{z}(z, F)$

\section{CONCLUSION}

In this paper, the overall qualitative image of the stressing issue of the rectangular prismatic piezoceramic cantilever with longitudinal polarisation and with electrode coatings is observed.

Conclusions derived from numerically obtained bi-parametric surfaces of state using the Matlab software package are:

- Fig. 2 shows that the value of componential displacement is equal to zero for $z=0$, which is to be expected based on equation (14). On the other hand, the oscillation node occurs at $x=0.0281 \mathrm{~m}$ for the selected dimensions of the observed piezoceramic PZT4 cantilever. At that spot, in the observed regime, it is possible to, for example, fix the cantilever without affecting its oscillations.

- Fig. 3 shows the fulfilled conditions of rigid clamping for the front surface $x=l$ $(w=0)$.

- For the electric potential $\psi$ (see Fig. 4) and the electric field $E_{z}$ (see Fig. 7) an almost sine law of change along the $z$-axis of the cantilever is justified, where the maximal difference of electric potential occurs at spots where $E_{z}=0$ (for $z=$ $\pm 0.0058 \mathrm{~m}$ ), and vice versa for $z= \pm h / 2$. The electric field $E_{z}=E_{z}\left(z, U_{0}\right)$ (see Fig. 8) has a characteristic half-cylinder spatial surface as well as the piezoelectric displacement $D_{z}$ (see Fig. 10).

- Bi-parametric surfaces of dilatation $\varepsilon_{x}$ (see Fig. 5) have extreme values on the frontal surface $x=l$. For bi-parametric surfaces of slide $\gamma_{x y}$ (see Fig. 6), a linear dependence is justified. 
- Piezoelectric displacement $D_{x}$ (see Fig. 9) has a characteristic saddle-shaped spatial surface.

- If the velocity of the longitudinal wave propagation in the piezoceramic electro-elastic rod is taken into account, which is $c=1 / \sqrt{\rho \varepsilon_{33}^{E}\left(1-k_{33}^{2}\right)}$, as well as the coefficient of electromechanical static relation in the longitudinal direction $k_{33}^{2}=b_{33}^{2} / \varepsilon_{33}^{E} d_{33}^{\sigma}$, one may get a resonant oscillation frequency of $174.28 \mathrm{kHz}$.

This kind of analysis enables the prediction of the characteristics of piezoceramic cantilevers (as actuators or sensors) with the presented configuration when designing piezoceramic devices. The measurement of mechanical displacements of the cantilever will be the subject of further research.

Acknowledgement: This work has been supported by the Ministry of Education, Science and Technological Development of the Republic of Serbia.

\section{REFERENCES}

[1] I. Jovanović, Lj. Perić, U. Jovanović, D. Mančić, "Analysis of Longitudinal Oscillations of Piezoceramic Cantilever with Electrode Coatings," in Proceedings of 19th International Conference on Thermal Science and Engineering of Serbia SimTerm, Sokobanja, Serbia, pp. 801-807, 2019.

[2] T. Nikolić, M. Stojčev, G. Nikolić, G. Jovanović, "Energy harvesting techniques in wireless sensor networks," Facta Universitatis, Series: Automatic Control and Robotics, vol. 17, no 2, pp. 117-142, 2018. [Online]. Available: https://doi.org/10.22190/FUACR1802117N

[3] H. Fu, G. Chen, N. Bai, "Electrode coverage optimization for piezoelectric energy harvesting from tip excitation," Sensors, vol. 18, no 3, paper no. 804, 2018. [Online]. Available: http://dx.doi.org/10.3390/ s18030804.

[4] T. Soyata, L. Copeland, W. Heinzelman, "RF Energy Harvesting for Embedded Systems: A Survey of Tradeoffs and Methodology," IEEE Circuits and Systems Magazine, vol. 16, no. 1, pp. 22-57, 2016. [Online]. Available: http://dx.doi.org/10.1109/MCAS.2015.2510198.

[5] C. R. Bowen, H. A. Kim, P. M. Weaver, S. Dunn, "Piezoelectric and ferroelectric materials and structures for energy harvesting applications," Energy Environ. Sci., vol. 7, no. 1, pp. 25-44, 2014. [Online]. Available: http://dx.doi.org/10.1039/C3EE42454E

[6] E. Macho-Stadler, M. J. Elejalde-García, R. Llanos-Vázquez, "Oscillations of end loaded cantilever beams," Eur. J. Phys., vol. 36, paper no. 055007, 2015. [Online]. Available: http://dx.doi.org/10.1088/0143-0807/36/ $5 / 055007$

[7] I. Jovanović, D. Mančić, V. Paunović, M. Radmanović, V. V. Mitić, "Metal Rings and Discs Matlab/Simulink 3D Model for Ultrasonic Sandwich Transducer Design," Science of Sintering, vol. 44, no. 3, pp. 287-298, 2012. [Online]. Available: https://doi.org/10.2298/SOS1203287J

[8] I. Jovanović, D. Mančić, U. Jovanović, M. Prokić, "A 3D model of new composite ultrasonic transducer," Journal of Computational Electronics, vol. 16, no. 3, pp. 977-986, 2017. [Online]. Available: http://dx.doi.org/ 10.1007/s10825-017-1000-0

[9] I. Jovanović, Lj. Perić, U. Jovanović, D. Mančić, "Stressing issue of a piezoceramic sectional cylinder with a circular polarization," in Proceedings of 26th International Conference Noise and Vibration 2018, Niš, Serbia, pp. $155-159,2018$.

[10] D. Rašković, Teorija elastičnosti (in serbian), Beograd: Naučna knjiga, 1985.

[11] Lj. Perić, Spregnuti tenzori stanja piezoelektričnih materijala (in Serbian), PhD thesis, University of Niš, Faculty of Mechanical Engineering, 2004

[12] I. Jovanović, Lj. Perić, U. Jovanović, D. Mančić, "Stressing Issue of a Piezoceramic Cantilever with Electrode Coatings and Transversal Polarization," Facta Universitatis, Series: Working and Living Environmental Protection, vol. 12, no. 1, pp. 123-137, 2015. [Online]. Available: http://casopisi.junis.ni.ac.rs/index.php/ FUWorkLivEnvProt/article/view/653

[13] Technical Publication TP-226, Morgan Electro Ceramics [Online]. Available: http://www.ultrasonicresonators.org/misc/references/articles/Berlincourt_\%27Properties_of_Morgan_Electro_Ceramic_Ceramics\% 27_(Morgan_Technical_Publication_TP-226).pdf [Accessed on August 2020]. 